\title{
Modeling of thermally stimulated depolarization current (TSDC) using dipole-dipole interaction concept
}

\author{
A E KOTP \\ Physics Department, Faculty of Science, Mansoura University, Mansoura, Egypt
}

MS received 2 August 2007; revised 22 December 2010

\begin{abstract}
The study of thermally stimulated depolarization current (TSDC) using the dipole-dipole interaction model is described in this work. The dipole-dipole interaction model (DDIM) determines the TSDC peak successfully since it gives significant peak parameters (i.e. activation energy $(E)$ and pre-exponential factor $\left(\tau_{0}\right)$ ) in addition to the dipole-dipole interaction strength parameter $\left(d_{i}\right)$. Application of this model to study the peak parameters of some polymeric systems is presented.
\end{abstract}

Keywords. Dipole-dipole interaction; relaxation; modeling; TSDC; activation energy; PVC; ABS.

\section{Introduction}

The study of molecular motion in polymers is gaining attention. With the aid of modeling and experimentation, we take an insight into the molecular motion through the study of relaxation processes which take place inside the polymers. In these studies, often two techniques are used: the first is dielectric spectroscopy (DS), and the other, the thermally stimulated depolarization current (TSDC).

The polymer as a modeling system is a very large and complicated molecular system which uses the phenomenology of the relaxation process (i.e. without considering the objective reality of the process).

In this work, the model depends on the polarization phenomena which takes place in the relaxation process during TSDC experiments, in addition to the dipole-dipole interaction concept which takes place between the oriented dipoles during the orientation process. The model provides data to investigate the contribution of dipole-dipole interaction to the whole TSDC curve.

\section{Model theory}

The mathematical model made by assuming the contribution of the dipole-dipole interaction is affecting only the width of TSDC peak, which leads to assuming further that the error due to the dipole-dipole interaction in the TSDC curve is distributed normally on the curve. By considering the Gaussian distribution according to the local limit theory (Gnedenko

(am2000de@yahoo.com)
1988), it is found that the error due to the dipole-dipole interaction is given by a correction factor:

$$
\begin{aligned}
C_{\mathrm{f}}= & \frac{1}{d_{\mathrm{i}} \sqrt{\pi}} \int_{-\infty}^{\infty} \exp \left(\frac{-E-E_{0}}{k T}\right) \exp \left(\frac{\left(-E-E_{0}\right)^{2}}{d_{\mathrm{i}}}\right) \\
& \exp \left(\frac{-1}{\beta} \int_{T_{1}}^{T_{2}}\left(\frac{1}{\tau(T)}\right)\left(\frac{1}{\tau_{E_{0}}(T)}\right) \mathrm{d} T\right)
\end{aligned}
$$

where $E_{0}$ is the centre of the energy distribution, $d_{\mathrm{i}}$ its width, $\beta=\mathrm{d} T / \mathrm{d} t$ the heating rate, $E$ the activation energy, and $\tau(T)$ the relaxation model.

Multiplying the correction factor $\left(C_{\mathrm{f}}\right)$ with the TSDC curve model gives

$$
I(T)=\frac{\left(A P_{0}\right)}{\tau} \exp \left(\left(\frac{-1}{\beta}\right) \int_{T_{1}}^{T_{2}}\left(\frac{1}{\tau} \mathrm{d} T\right)\right),
$$

where $A$ is sample area, $P_{0}$ the initial polarization and $\tau(T)$ the relaxation model used. Equation (2) gives the corrected TSDC curve as

$$
I_{\text {corr }}(T)=C_{\mathrm{f}}^{*} I(T) .
$$

The theoretical correction factor $\left(C_{\mathrm{f}}\right)$ is studied very intensively in order to get a complete picture about the correction factor behaviour along with all variables and constants using MathCAD. After the analysis of the correction factor $\left(C_{\mathrm{f}}\right)$, a Fortran 95 program was made in order to compute (3).

The improper integral over the energy $(E)$ was approximated (Myskis 1978), and the integral over $T$ was calculated using the Simpson Composite method (Burden and Faires 1989), where it is found to be more accurate for the exponential function. The fitting was made using the $\chi^{2}$ technique 
between the experimental data and the computed data with an error $\varepsilon=1 \times 10^{-5}$.

\section{Model development}

The dipole-dipole interaction (DDI) model was developed using two approaches depending on the relaxation model used.

\subsection{Using Arrhenius relaxation model}

The dipole-dipole interaction model was proposed to investigate use of the Arrhenius relaxation model, $\tau(T)$, given by the well known equation

$$
\tau(T)=\tau_{0} \exp \left(\frac{E}{k T}\right)
$$

Using this equation in the correction factor, we have

$$
\begin{aligned}
C_{\mathrm{f}}= & \frac{1}{\left(d_{\mathrm{i}} \sqrt{\pi}\right)} \int_{-\infty}^{\infty} \exp \left(\frac{\left(-E-E_{0}\right)}{k T}\right) \exp \left(\frac{\left(-E-E_{0}\right)^{2}}{d_{\mathrm{i}}}\right) \\
& \exp \left[\frac{-1}{\beta} \int_{T_{1}}^{T_{2}} \exp \left(\frac{-E}{k T}\right) \exp \left(\frac{-E_{0}}{(k T)}\right) \mathrm{d} T\right] \mathrm{d} E .
\end{aligned}
$$

Also we can do the same for the initial current equation to become

$$
I(T)=\frac{\left(A P_{0}\right)}{\tau_{0}} \exp \left(\frac{-E}{k T}\right) \exp \left(\frac{-1}{\left(\beta \tau_{0}\right)} \int_{T_{1}}^{T_{2}} \exp \left(\frac{-E}{k T}\right) \mathrm{d} T\right) .
$$

\subsection{Using Vogel-Fulcher-Tammann relaxation model}

We use the dipole-dipole interaction model to investigate use of the Vogel-Fulcher-Tammann relaxation model, $\tau(T)$, given by the well known equation

$$
\tau(T)=\tau_{0} \exp \left(\frac{E}{k\left(T-T_{0}\right)}\right) \text {. }
$$

Then applying this equation again in the correction factor equation we have

$$
\begin{gathered}
C_{\mathrm{f}}=\frac{1}{\left(d_{\mathrm{i}} \sqrt{\pi}\right)} \int_{-\infty}^{\infty} \exp \left(\frac{\left(-E-E_{0}\right)}{k T}\right) \exp \left(\frac{\left(-E-E_{0}\right)^{2}}{d_{\mathrm{i}}}\right) \\
\exp \left[\frac{-1}{\beta} \int_{T_{1}}^{T_{2}} \exp \left(\frac{-E}{\left(k\left(T-T_{0}\right)\right)}\right)\right. \\
\left.\quad \exp \left(\frac{-E_{0}}{\left(k\left(T-T_{0}\right)\right)}\right) \mathrm{d} T\right] \mathrm{d} E
\end{gathered}
$$

and the current equation becomes

$$
\begin{aligned}
I(T)= & \frac{\left(A P_{0}\right)}{\tau_{0}} \exp \left(\frac{-E}{\left(k\left(T-T_{0}\right)\right)}\right) \\
& \exp \left(\frac{-1}{\left(\beta \tau_{0}\right)} \int_{T_{1}}^{T_{2}} \exp \left(\frac{-E}{\left(k\left(T-T_{0}\right)\right)}\right) \mathrm{d} T\right) .
\end{aligned}
$$

Using (5)-(6) and (8)-(9), we have two models to work with: the first is the dipole-dipole interaction model using Arrhenius relaxation model (we shall call it DDIA) and the second is the dipole-dipole interaction model using Vogel-Fulcher-Tammann relaxation model (we shall call it DDIVFT).

\section{Results and discussion}

In order to investigate the dipole-dipole interaction model in the Arrhenius mode DDIA applicability, experimental data peaks obtained from poly(vinyl chloride) (PVC) and acrylonitrile-butadiene-styrene (ABS) using the thermal sampling technique were used. Furthermore, to compare the model, calculated peak parameters (i.e. the energy $(E)$ and pre-exponential factor $\left.\left(\tau_{0}\right)\right)$, two known peak shape methods were used: (i) the initial rise method (IR) (Garlick and Gibson 1948; Hoogenstraaten 1958), (ii) the half width method (HW) (Chen 1969) in addition to the Cowell and Woods (1967) analysis.

In order to assess the quality of fitting of DDIA model with the experimental data, the calculated TSDC points was plotted against the experimental data. Furthermore, the DDIA model fitting was compared with the Cowell-Wood method. The fitting of the experimental and calculated data using Cowell-Wood method shows a poor fitting specially in the high- and low-temperature sides. The fitting of experimental and calculated data using DDIA model shows that the fitting is much more correlated to the experimental data which indicates that the dipole-dipole interaction affects the TSDC current peak, which was neglected before in the analysis of the TSDC current peak.

Table 1 shows the dipole-dipole interaction model in Arrhenius mode (DDIA) calculated peak parameters $(E)$, $\left(\tau_{0}\right)$, in addition to dipole-dipole interaction strength parameter $\left(d_{\mathrm{i}}\right)$. Both the activation energy $(E)$ and dipole-dipole interaction strength $\left(d_{\mathrm{i}}\right)$ are introduced as $(\mathrm{kj} / \mathrm{mole})$ and the pre-exponential factor $\left(\tau_{0}\right)$ is introduced in seconds for PVC polymer material.

Table 2 shows the dipole-dipole interaction model in Arrhenius mode (DDIA) calculated peak parameters $(E)$, $\left(\tau_{0}\right)$, in addition to dipole-dipole interaction strength parameter $\left(d_{\mathrm{i}}\right)$. Both the activation energy $(E)$ and dipole-dipole interaction strength $\left(d_{\mathrm{i}}\right)$ are introduced as $(\mathrm{kj} / \mathrm{mole})$ and the pre-exponential factor $\left(\tau_{0}\right)$ is introduced in seconds for ABS terpolymer. 

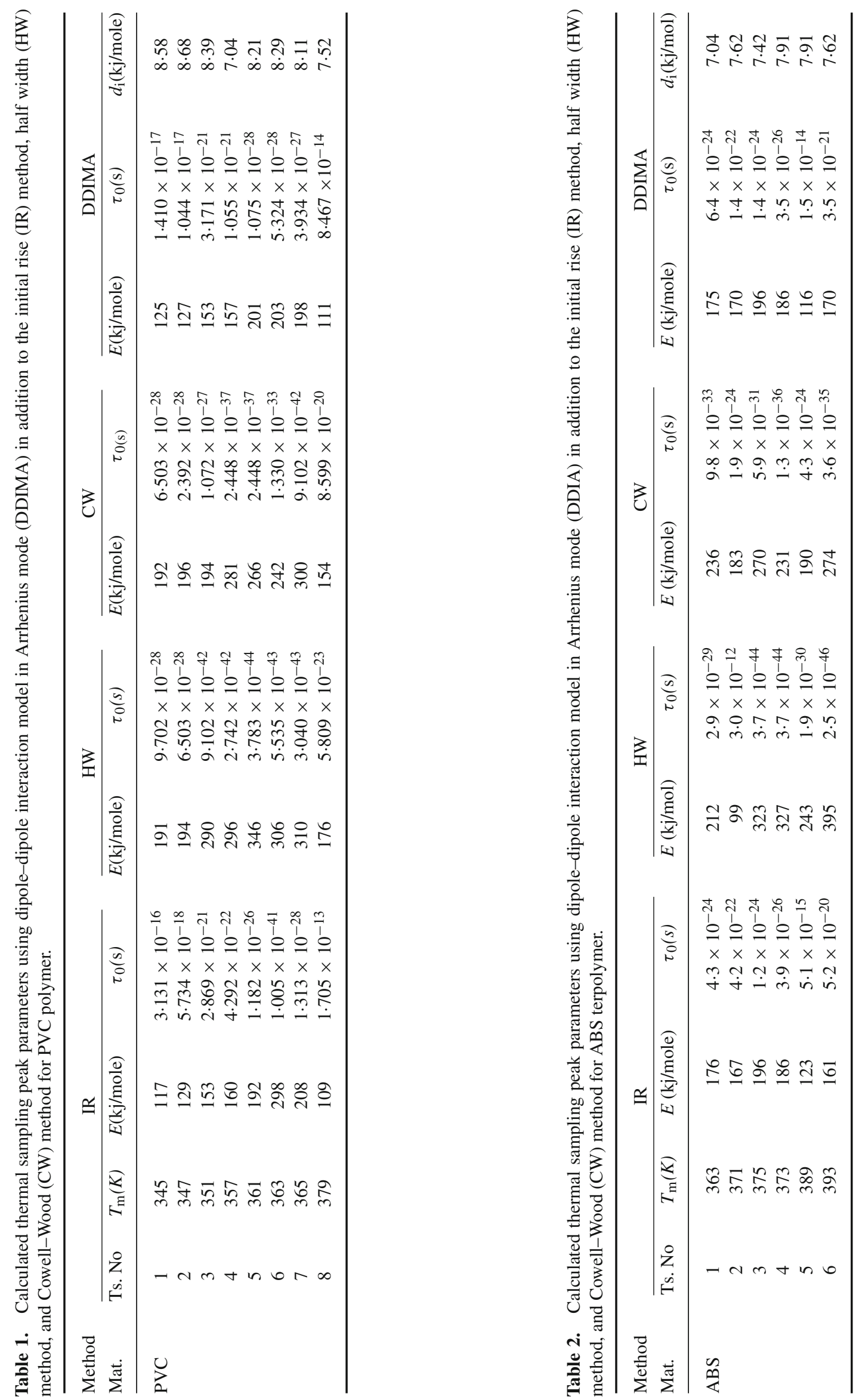


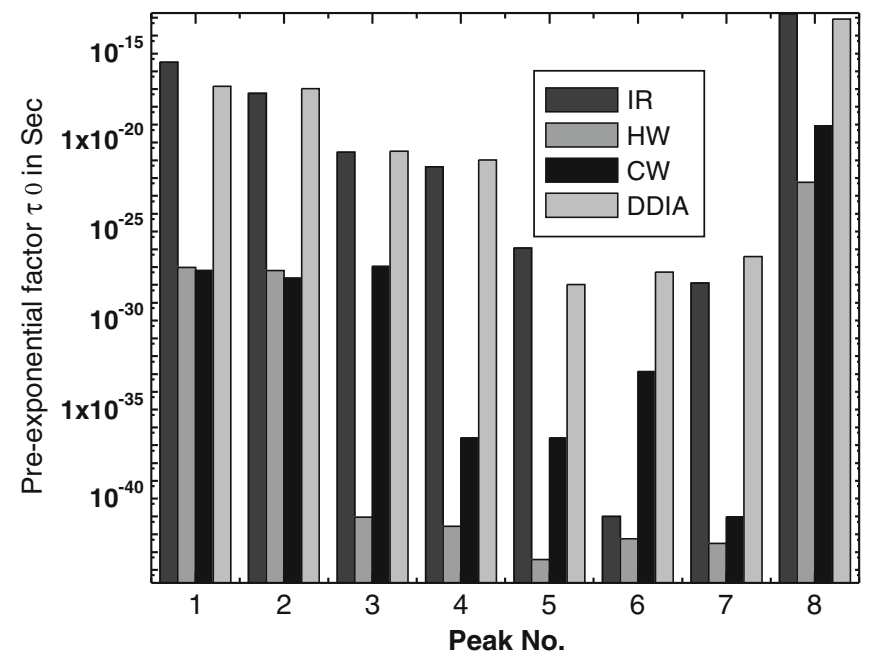

Figure 1. Pre-exponential values obtained using three different methods: IR, HW, CW, and DDIA model for the PVC.

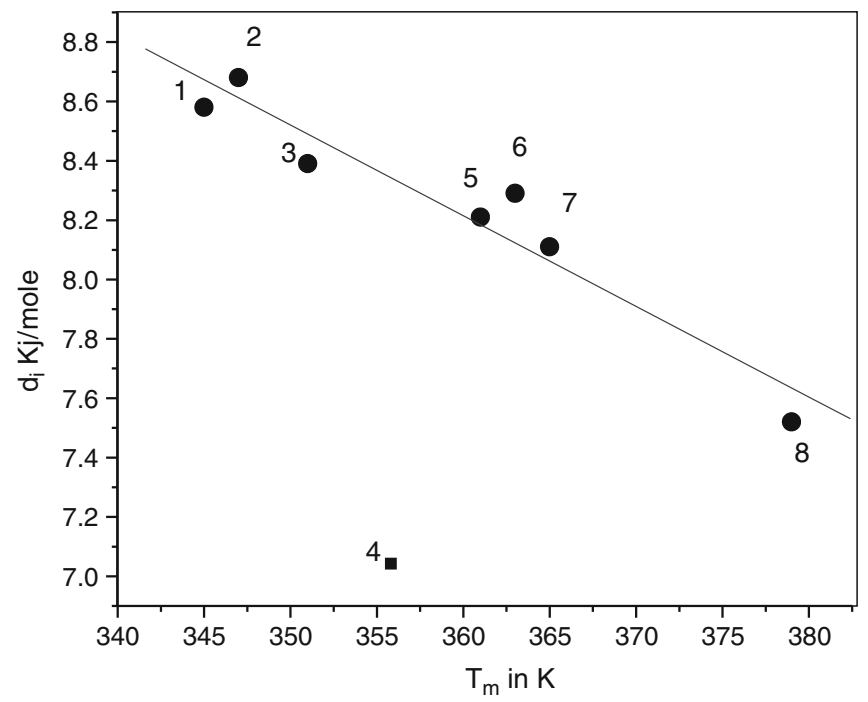

Figure 2. Relationship between calculated dipole-dipole interaction parameter $\left(d_{\mathrm{i}}\right)$ and maximum temperature $\left(T_{\mathrm{m}}\right)$ for PVC.

The model-calculated results show that the DDIA data give significant figures for the pre-exponential factor $\left(\tau_{0}\right)$, whereas the initial rise method, the half-width and CowellWood methods give non-significant figures. This indicates that the DDIA model gives more correction to the calculated relaxation time. Furthermore, the calculated activation energy $\left(E_{\mathrm{a}}\right)$ using the DDIA model is less in magnitude than those calculated using other methods which gives more significant values for the activation energy.

Figure 1 shows a comparison of the calculated preexponential factor $\left(\tau_{0}\right)$ using four different methods, IR, HW, $\mathrm{CW}$, and DDIA, respectively. In this figure, it is found that the pre-exponential factors calculated using the IR method and DDIA are closely correlated except in TS peak no. 6 ,

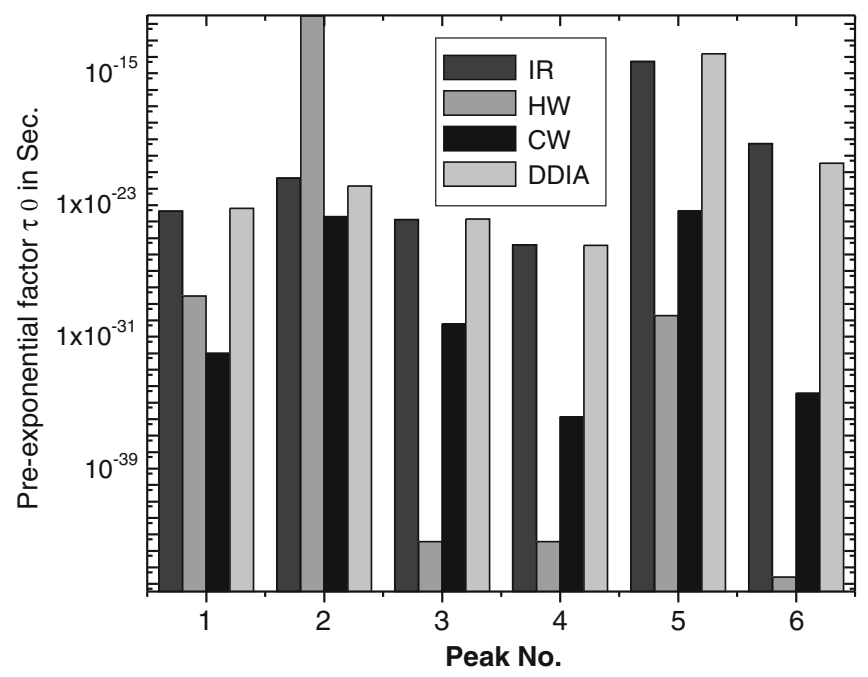

Figure 3. Pre-exponential values obtained using three different methods: IR, HW, CW, and DDIA model for ABS.

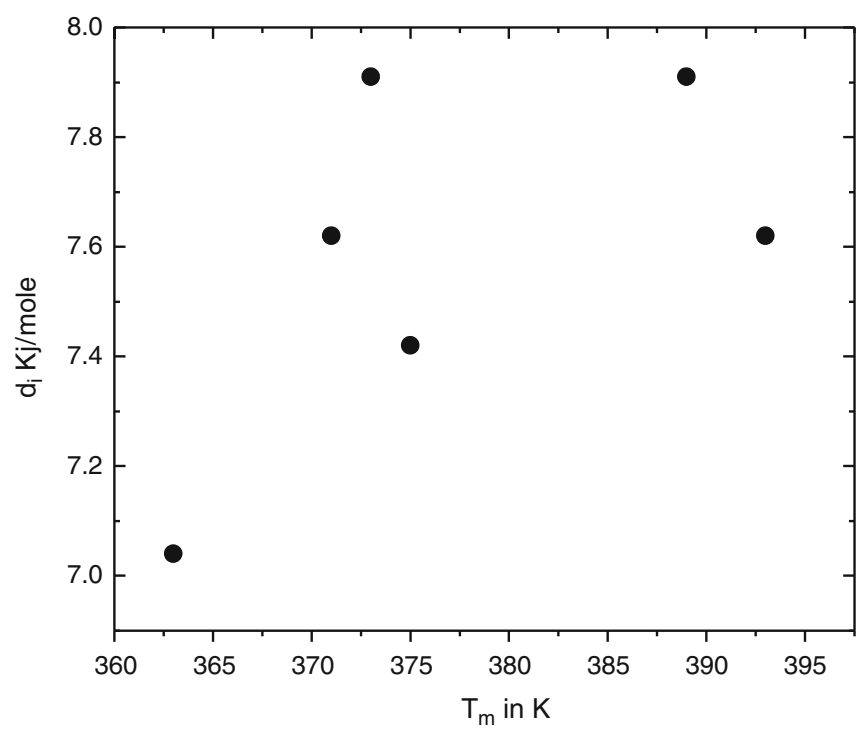

Figure 4. Relationship between calculated dipole-dipole interaction parameter $\left(d_{\mathrm{i}}\right)$ and maximum temperature $\left(T_{\mathrm{m}}\right)$ for ABS.

the calculated activation energy using the IR method gives a very low value of $1 \times 10^{-41} \mathrm{~s}$, whereas, DDIA model gives a more significant value of $5.3 \times 10^{-28} \mathrm{~s}$. These results indicate that the DDIA model correct the errors in the peak shape methods (IR, HW) and also gives more correct values than the CW method. On the other hand, it is found that the CW method is much correlated to the HW method, except in TS peaks 3 and 5 where they give more values.

Figure 2 shows the dipole-dipole interaction parameter $\left(d_{\mathrm{i}}\right)$ calculated using the DDIA model for PVC against maximum temperature $\left(T_{\mathrm{m}}\right)$ which shows that this parameter depends linearly, except TS peak no. 4, on the temperature which may be related to the homogeneity of this polymer. 
Figure 3 shows a comparison of the calculated preexponential factor $\left(\tau_{0}\right)$ using four different methods, IR, HW, $\mathrm{CW}$, and DDIA, respectively. In this figure, it is found that the pre-exponential factors calculated using the IR method and DDIA are closely correlated in all peaks.

Figure 4 shows the dipole-dipole interaction parameter $\left(d_{\mathrm{i}}\right)$ calculated using the DDIA model for the ABS against maximum temperature $\left(T_{\mathrm{m}}\right)$ which shows that this parameter has no regular dependence on the temperature which may be related to the heterogeneity nature of this polymer.

These results indicate that the DDIA model corrects the errors in the peak shape methods, HW and also gives more correct values than the $\mathrm{CW}$ method. On the other hand, it is found that the $\mathrm{CW}$ method is much correlated to the HW method.

\section{Conclusions}

The dipole-dipole interaction model corrects the difference in the high and low temperature sides between experimental and calculated data using the CW method. This means that the dipole-dipole interaction affects the TSDC peak by increasing its width.
The dipole-dipole interaction model in Arrhenius mode DDIA gives more significant peak parameter values than other methods used especially in the calculation of the pre-exponential factor $\left(\tau_{0}\right)$.

Using the dipole-dipole interaction model in Arrhenius mode DDIA can lead to computation of the dipole-dipole interaction parameter $\left(d_{\mathrm{i}}\right)$ which gives an indication of the magnitude of this parameter in polymers.

The relationship of the newly calculated dipole-dipole interaction parameter $d_{\mathrm{i}}$ and the maximum temperature may indicate the homogeneity or heterogeneity of the polymer system.

\section{References}

Burden R L and Faires J D 1989 Numerical analysis (Boston: Kent) 4th ed. p. 451

Chen R 1969 Appl. Phys. 402

Cowell T A T and Woods J 1967 Br. J. Appl. Phys. 181045

Garlick B J and Gibson A F 1948 Proc. Phys. Soc. 60574

Gnedenko B 1988 Theory of probabilities (Moscow: Mir Pub.) p. 85

Hoogenstraaten W 1958 Philips Res. Rep. 13515

Myskis A D 1978 Introductory mathematics for engineers (Moscow: Mir Pub.) p. 454 